\title{
Activités
}

15-1 | 2018

Mettre en scène l'activité : les nouvelles figures de la simulation

\section{Simuler pour changer la conception et les rapports sociaux de conception du travail : un commentaire sur les dispositifs de simulation de l'activité dans les organisations}

Simulating to change the design and social relations of work design:

a commentary on activity simulation devices in organizations

Pascal Ughetto

\section{OpenEdition}

Édition électronique

URL : http://journals.openedition.org/activites/3101

DOI : 10.4000/activites.3101

ISSN : 1765-2723

Éditeur

ARPACT - Association Recherches et Pratiques sur les ACTivités

Référence électronique

Pascal Ughetto, « Simuler pour changer la conception et les rapports sociaux de conception du travail : un commentaire sur les dispositifs de simulation de l'activité dans les organisations ", Activités [En ligne], 15-1 | 2018, mis en ligne le 15 avril 2018, consulté le 19 avril 2019. URL : http:// journals.openedition.org/activites/3101 ; DOI : 10.4000/activites.3101

Ce document a été généré automatiquement le 19 avril 2019

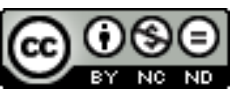

Activités est mis à disposition selon les termes de la licence Creative Commons Attribution - Pas d'Utilisation Commerciale - Pas de Modification 4.0 International. 


\title{
Simuler pour changer la conception et les rapports sociaux de conception du travail : un commentaire sur les dispositifs de simulation de l'activité dans les organisations
}

\author{
Simulating to change the design and social relations of work design:
} a commentary on activity simulation devices in organizations

Pascal Ughetto

\section{NOTE DE L'ÉDITEUR}

Article soumis le 25/02/2018, accepté le 15/03/2018

\section{Introduction}

Le présent dossier expose divers argumentaires en faveur de l'utilisation de méthodes de simulation dans des contextes domestiques aussi bien que d'organisations et présente une variété de dispositifs. Convoquer le point de vue de l'activité est une invitation pressante que des chercheurs en sciences sociales et des praticiens adressent très fréquemment aux concepteurs, tant pour le travail (technologies, organisation, outils de gestion) que pour des objets ou techniques de la vie quotidienne. Ces chercheurs et praticiens leur reprochent de faire reposer la conception sur des modélisations abstraites et décontextualisées, méconnaissant les situations, les contraintes rencontrées et les 
finalités poursuivies au sein de celles-ci, ou encore les enjeux que se donnent les personnes et la façon dont elles peuvent se saisir des ressources de l'environnement. De fait, dans la conception des cadres à l'intérieur desquels s'exerce le travail en organisation ou de ceux qui s'imposent dans d'autres sphères de la vie sociale - par exemple, le logement dans lequel vit une famille - les entités - et en particulier les êtres humains - ne se bornent pas à réagir de façon anticipable et en vertu de certaines règles ou lois de comportement, de telle sorte qu'il suffirait que les cadres soient parfaitement pensés pour que de «bonnes pratiques» en découlent. Tout passe, au contraire, par l'intermédiaire actif d'activités, déployées par des sujets, eux-mêmes guidés par les finalités qu'ils cherchent à atteindre, les interprétations qu'ils se font des contextes, les contraintes qu'ils gèrent, un équipement de l'action que leur offre l'environnement et dont ils peuvent plus ou moins se saisir.

2 Mais comment fait-on pour convoquer le point de vue de l'activité ? Comment fait-on pour que celui-ci irrigue réellement et durablement un processus de conception? Ce sont ce que s'emploient à réaliser les approches qui s'intéressent à la simulation. Dans le cadre de ce commentaire, je me limiterai à la conception du travail en organisation. À son propos, je défendrai que l'intérêt de la simulation réside dans sa capacité à instrumenter les rapports sociaux de conception de façon plus contraignante que d'habitude pour les organisateurs, et à accorder plus authentiquement, que ce n'est généralement le cas, une place au travail.

\section{Pourquoi les approches de simulation de l'activité aujourd'hui ?}

\subsection{Simuler pour contraindre à la prise en compte de l'activité}

3 La simulation de l'activité dévoile d'autant mieux son intérêt qu'on la replace dans un double cadre : celui des invitations régulièrement faites par les chercheurs en direction des responsables d'entreprises ou d'administrations à privilégier une conception du travail qui intègre les préoccupations et le point de vue de l'activité (Guérin, \& al., 1997) ; et celui des évolutions actuelles, dans les discours et/ou les pratiques, en matière de modes de conception, passant par la mobilisation des utilisateurs et prenant la forme de boucles courtes de rétroaction.

4 En premier lieu, en effet, les dernières années ont vu se multiplier les invitations adressées par les chercheurs aux responsables d'entreprises ou d'administrations à privilégier une conception du travail qui intègre les préoccupations et le point de vue de l'activité, c'est-à-dire qui tienne compte du décalage entre le prescrit et le réel et se préoccupe des contraintes et ressources de l'activité (Béguin, \& Cerf, 2004). Mais la constance de ces invitations n'a d'équivalent que la difficulté des responsables en charge de la conception et du déploiement de nouveaux outils techniques, organisations, dispositifs de gestion, à se figurer de quoi il est question. Et, par ailleurs, leur difficulté à mettre en œuvre ce point de vue de l'activité sans perdre de vue les impératifs d'organisation; ou, pour dire les choses ouvertement, de contrôle. Les chercheurs ou les intervenants pleinement convaincus que la différence entre le travail prescrit et le travail réel est une donnée puissante s'étonnent volontiers que les organisateurs persistent à ignorer l'activité. Tenir compte de celle-ci devrait, à leurs yeux, être frappé au coin du bon sens. En réalité, dès lors qu'elle doit assurer une production à grande échelle, 
garantir une qualité stable, tenir des promesses avec une certaine régularité, une organisation développe des préoccupations de contrôle : de ses approvisionnements, de la fiabilité et du rendement des matériels, mais aussi des personnels. Bien qu'il s'agisse de travail humain, l'idéal de prévisibilité, de fiabilité et de constance se fait valoir. L'activité complique le travail de fiabilisation et en faire l'économie s'impose facilement.

5 Seconde dimension de l'actualité de la simulation, les évolutions ont considérablement renforcé également, dans les discours et/ou les pratiques relatives aux modes de conception, le sentiment que tout processus de conception devrait reposer sur la mobilisation des utilisateurs, des interactions serrées avec eux, voire des boucles courtes de rétroaction. Il s'agit ici des invitations pressantes, de la part de divers milieux professionnels, cercles de réflexion, consultants, à voir les entreprises se tourner vers des organisations fondées sur l'autonomie, le mode agile, des labs, des hackathons (Trupia, 2016), etc. Toutes ces formules interrogent la verticalité du fonctionnement des organisations, les délais de prises de décision, l'expression désincarnée et machinique des process et des circuits de décision, et postulent ou déclarent l'intolérance fondamentale des nouvelles générations à l'égard d'interactions avec la ligne managériale où il est difficile de faire valoir à la fois un point de vue et des réalités pratiques tangibles. Toutes postulent qu'on ne peut plus continuer à ignorer des formes de rejet de ce qui, dans les organisations contemporaines, entrave l'envie de faire, l'effort pour faire, la recherche par les individus contemporains de la possibilité de se retrouver dans ce qu'ils font (Crawford, 2009 ; Flichy, 2017).

6 À ces deux éléments de contexte, on pourrait ajouter la place croissante de l'expérimentation comme méthode de déploiement des politiques publiques autant que des politiques d'entreprises ou d'administrations (voire en recherche : Banerjee, \& Duflo, 2009). Autrement dit, l'idée qu'il faut tester à une échelle réduite, constater pragmatiquement ce qui fonctionne et ce qui fait moins ses preuves, avant de fixer et diffuser ensuite, en fonction des résultats.

\subsection{Pouvoir discuter authentiquement de la réalité}

7 Le point commun de tout cela est la question des épreuves de réalité, du renvoi à la réalité, de la confrontation au réel, et de la possibilité, entravée ou favorisée, de créer de la discussion sur cette base. Quelles sont les conditions pour qu'une discussion émerge et s'entretienne autour de la façon dont, dans une réalité concrète, située, les difficultés les plus ordinaires se manifestent, l'intelligence est mise à l'épreuve, des dispositifs soutiennent les stratégies d'activité ou les contrarient? De ces possibilités d'en discuter authentiquement dépend que le faire puisse prendre son sens, que des envies de faire se développent ou que des entraves au faire se manifestent. Un dispositif, une ingénierie, sont nécessaires pour que cette discussion se forme et s'organise. Les dispositifs de simulation de l'activité créent des possibilités de laisser voir, faire sentir, faire éprouver, faire constater, voire faire reconnaître.

8 En cela, on pourrait également établir un lien avec la place que prennent des dispositifs d'apparence très simple mais généralement salués par ceux qui en bénéficient et que les organisations nomment fréquemment des "Vis ma vie", opérations consistant à permettre aux membres d'un service d'aller passer une journée dans un service avec lequel ils ont des interactions constantes sans jamais les avoir vu fonctionner dans leurs locaux. 
Utiliser la simulation comme un moyen pour intégrer les préoccupations d'activité dans la conception du travail et des cadres de travail est loin d'être une question nouvelle, en tout cas pour l'ergonomie, ce que rappelle L. Van Belleghem (2012 et ce numéro). De même que, en gestion, pour l'implantation d'ateliers ou pour divers processus de conception. En quoi, néanmoins, la simulation de l'activité participe-t-elle d'une évolution possible des rapports sociaux de conception des objectifs du travail et des cadres de ce travail et du rapport à la prescription? Comment cela interroge-t-il les acteurs de la conception du travail et de la prescription? Qu'est-ce que cela dit du contrôle organisationnel ? On peut notamment soutenir que cela expose les concepteurs à une diversification des scénarios sans contrôle a priori et donc à une plus grande ouverture et indétermination des processus de conception. Cela les oblige à entrer dans la co-conception d'une organisation dont le processus n'est pas totalement «ficelé ». Cela les force à relâcher le contrôle immédiat.

\section{La simulation de l'activité dans l'écologie des méthodes de conduite du changement préconisant le participatif}

Alors même que les process ont accru leur domaine au cours des dernières années, jusqu'à subir de fortes critiques du point de vue de leur effet déréalisant pour le travail (Ginsbourger, 2010 ; Gomez, 2013), il n'est guère de changements qui n'aient été assortis de méthodes de gestion participative pour leur diffusion. Il n'est guère de nouvelle réalité technique, organisationnelle ou de gestion qui ne prévoit aujourd'hui sa « conduite » ou son "accompagnement du changement», avec parfois des services ou des individus dédiés.

11 C'est vis-à-vis des techniques de conduite du changement que la simulation de l'activité mérite d'être située. Courantes sont les démarches qui demandent aux intéressés de se projeter et de faire connaître leurs attentes : «Imaginez votre poste de travail », « De quel lieu avez-vous besoin?» Ces méthodes ont généralement un rendement décevant : au grand regret des animateurs, les salariés ne font guère de suggestions, peinent à imaginer quoi que ce soit de différent de l'existant, attendent de voir pour pouvoir dire.

12 Cela alors qu'il n'est guère de changement, également, qui ne soit introduit sans une profession de foi des services centraux et personnes qui l'animent pour certifier qu'ils veulent avant tout que les salariés qui en seront les utilisateurs effectifs trouvent le nouveau dispositif utile, qu'il leur facilite la vie, qu'il soit agréable au plan ergonomique.

Ces méthodes et ces professions de foi ne parviennent cependant pas à dissimuler l'inquiétude des auteurs ou pilotes du changement quant aux "résistances», aux comportements récalcitrants ou peu coopératifs, à la faible mobilisation, à la morosité diffuse ou à la critique ouverte. De fait, ces démarches n'entament guère le sentiment des salariés que le changement est, quoi qu'il en soit, issu de personnages éloignés du terrain et ne connaissant pas l'activité, incapables de prendre en compte cette dernière ou non désireux de le faire. Les décideurs en appellent à la participation tout en craignant la préférence des intéressés pour le maintien dans leur «zone de confort », tandis que les salariés restent dans l'expectative quant à l'effectivité d'une co-construction. Le dialogue de sourds peut se prolonger avec des membres du management qui, aux premières 
plaintes qui s'exprimeront plus tard, opposeront aux intéressés qu'il leur avait été demandé de s'exprimer et qu'ils n'ont pas su ou pas voulu le faire.

La simulation de l'activité, vais-je défendre, est de nature à desserrer cet étau, en forçant les décideurs à considérer plus authentiquement les préoccupations d'activité des salariés et, dès lors, en changeant les conditions du jeu pour ces derniers.

\section{Faire éprouver pour obliger à se figurer et pour faire valoir : instrumenter les politiques de reconnaissance}

\subsection{Ce que permet la simulation}

Quelle est la spécificité de la simulation de l'activité ? Quittons dans un premier temps cette dernière et prenons l'exemple d'un exercice de simulation qui vise moins à concevoir qu'à s'entraîner, l'exercice de simulation d'une crue de la Seine qui s'est tenu en 2016, sous le nom de "Sequana". Pendant deux semaines, sur le périmètre de l'Ile-deFrance, il s'agissait de simuler une montée des eaux, puis une décrue, et de demander aux institutions, organisations publiques, privées ou autres, potentiellement concernées de jouer leur rôle à travers certains de leurs agents (November, \& Créton-Cazanave, dir., $2017^{1}$ ). Au plus profond, l'exercice est destiné à chacun à se préparer et, ce faisant, à participer à la construction d'une planification. Face à l'incertitude, voire à l'inimaginable (ibid., p. 37), il faut être capable de faire face et d'éviter les actions désordonnées. Les effets recherchés sont multiples (ibid., p. 45) : le fait pour les agents concernés de devenir sensibles à ce qui pourrait survenir en l'ayant éprouvé; développer une meilleure compréhension des flux d'information et des mécanismes de leur circulation et des interdépendances qui lient ces agents à d'autres; notamment, de mieux se figurer ces autres en les ayant vus à l'œuvre, d'avoir une information sur leur existence, leur champ d'action, leurs logiques d'action, leurs contraintes, leurs enjeux ; mais aussi apprendre, tester, une fois de plus éprouver, des coordinations possibles, leurs limites, leurs avantages, d'autres manières de faire, et envisager des formules aidant à réduire les dissonances, les divergences de points de vue, d'appréciation, de qualification des situations, etc. Et, sur ces bases, l'effet est aussi de les aider à s'approprier les procédures. L'enjeu est bien de construire une planification susceptible de se traduire dans des actes effectifs. Avant l'exercice, ceux-ci se heurtent à des obstacles qui contrarient le cheminement des esprits, la disposition de ces esprits à s'orienter vers ce que la planification exigerait d'eux si l'épisode survenait: l'incrédulité (la difficulté à se représenter la simple possibilité sérieuse de la crue, un antécédent comme celui de 1910 n'existant par exemple que très faiblement, à travers les seules cartes postales) (ibid., p. 75) est d'abord à surmonter pour que, ensuite, les esprits, en quelque sorte, pivotent : à partir du moment où les personnes se figurent les situations, elles peuvent se projeter dans ce qu'elles seraient amenées à faire et envisager les implications - pour elles-mêmes et pour les autres - de leurs conduites. Les standards attendus de comportement n'ont des chances d'être incorporés que dans la mesure où le détour est fait par cet effort pour les aider à envisager sérieusement une possibilité et par ce que celle-ci permet en termes de disponibilité des personnes pour incorporer, intégrer.

Faire éprouver permet également de réduire la difficulté cognitive à se représenter et à se projeter dans la complexité (d'acteurs, d'interactions, une dimension systémique, etc.) ( ibid., p. 80). Appréhendée sur le plan abstrait, cette complexité est de nature à décourager 
l'effort de compréhension et de maîtrise. Le concret permet, là encore, de rendre disponible pour envisager, en surmontant la réticence possible des personnes à entrer dans cette complexité.

17 Une ingénierie du jeu est nécessaire pour organiser une simulation qui, tout en étant simplificatrice, permet de produire ces effets que sont : éprouver, réaliser, concevoir, envisager avec sérieux, etc. Cette ingénierie repose sur la résolution de questions comme : quels temps de jeu; faut-il tronquer des situations, découper ce qui se manifesterait en réalité de manière intégrée, reconduire un même exercice pour que plusieurs participants aient l'occasion d'y intervenir (ibid., partie 2), quand bien même ces choix artificialisent les situations par rapport à celles qui seraient vécues dans la réalité? Tout cela peut constituer des conditions pour que les participants se projettent effectivement et acceptent d'envisager des choses qui comptent fondamentalement : à savoir ce que leur action implique pour les autres et pour les faits matériels.

\subsection{Déplacer les rapports sociaux de conception}

18 Dans cette perspective se dessine déjà ce qui devient central dans ce qui est évoqué au sein de ce dossier : faire jouer permet de faire « réaliser ». D’abord dans le sens de faire prendre conscience mais ensuite aussi dans le sens de contraindre un décideur qui, en théorie, aurait une position lui permettant de n'en tirer que modérément les conséquences, à prendre au sérieux ce que les salariés éprouvent dans l'activité. Dans les organisations, marquées par le rapport de subordination et l'exercice d'un pouvoir de direction, cela veut dire particulièrement obliger un acteur décisionnaire à voir ce dont il pourrait habituellement vouloir et pouvoir faire abstraction.

19 Ce qui semble particulièrement caractéristique de ce qui est exposé par plusieurs articles de ce dossier est que les dispositifs font faire l'expérience de ce qui pourrait se passer. Ils permettent d'éprouver et de faire éprouver : voilà ce qui est caractéristique. Ce simple fait est susceptible de provoquer des déplacements. Du côté des salariés et au niveau des processus d'itération entre l'abstrait et le concret et, par là même, des processus de figuration des réalités susceptibles d'advenir, la simulation évite l'abstraction de la demande «Imaginez votre poste de travail.» Ce faisant, elle provoque davantage la représentation de ce que cela engage et, par suite, engendre plus facilement une parole sur le projet et le travail. Cela aide les salariés à avoir un avis à exprimer, une opinion, à la faire connaître. Les déplacements sont appelés également du côté de la ligne managériale et au niveau de la perception et prise en considération par le management des arguments des subordonnés qui, habituellement, s'expriment couramment ainsi : "Ce sera difficile, ce ne sera pas possible ». Là où les membres du management - y compris par difficulté à se figurer la véracité du problème soulevé par les salariés et l'effectivité que ce problème pourrait conserver dans le futur dispositif - sont tentés de rejeter cette expression comme le signe d'une mauvaise volonté, d'une faible coopération à la conception ellemême, la simulation permet d'observer et mesurer cette effectivité. La simulation les oblige à accuser réception de la parole exprimée. Car la réalité habituelle des processus de conception du travail participatifs est telle que, même avec une ligne managériale sincère, le dialogue bute souvent sur le fait que les salariés ne participent pas assez, au goût des managers, tandis que ces salariés ont le sentiment que le jeu des managers n'est pas totalement sincère. La participation en actes a ses limites (Charles, 2016) 
20 Autrement dit, potentiellement, la simulation peut aider les salariés à se figurer les réalités futures et à se donner ainsi plus facilement un avis, pour mieux faire valoir cet avis (au sens de le faire entendre et d'en faire reconnaître la valeur, l'apport). Et, par ailleurs, cela arme les membres de la ligne managériale plus que les techniques participatives un peu creuses. Quand bien même cet avis serait essentiellement négatif (« Vous voyez bien que cela ne fonctionne pas»), les membres du management, qui sont habituellement tentés de rejeter cela en stigmatisant un « comportement négatif » simplement parce que cela constitue, dans leur activité, une entrave qui ne trouve pas de voie de dépassement évidente, peuvent ici plus facilement entendre un tel avis : il leur est, en effet, plus facile de partager le constat et d'imaginer une solution à proposer. Ils sont moins dépourvus, ils sont plus équipés. Cela modifie potentiellement le cours même de l'interaction.

21 Dès lors, en second lieu, cela déplace les conditions, les termes, du dialogue, en évitant le renvoi des salariés dans leurs buts: «Mais si ! Ce sera possible. Arrêtez d'être négatifs, proposez au lieu de critiquer » ou "C'est justement pour que cela devienne possible que l'on change; ce que vous nous opposez est précisément ce qui existait dans le fonctionnement actuel et que l'on veut combattre dans le nouveau dispositif ». Dans le dialogue "traditionnel », la discussion ne trouve pas de prise, ne s'arrête pas sur des éléments de réalité, et, par conséquent, peut finir par du discours contre du discours, une parole contre une parole, une croyance contre une croyance.

A contrario, l'activité est potentiellement un juge de paix. En ancrant la discussion dans la référence au réel, la simulation de cette activité ne dissout pas les désaccords, les divergences de points de vue, mais arrête potentiellement une partie de la dérive vers ce régime purement rhétorique.

La simulation est un moyen de tenir jusqu'au bout des considérations d'activité. C'est potentiellement un instrument de modification du dialogue managérial autour du travail. Le plus souvent, ce dialogue est dévitalisé par le fait que l'activité ne fait pas foi. L'enjeu de la simulation est de crédibiliser et, ce faisant, d'imposer les considérations d'activité, c'est-à-dire les enjeux, contraintes, ressources des situations. L'effet possible est que, pour une fois, l'acteur managérial se trouve réellement amené à envisager le dialogue, là où la limite du management participatif est classiquement d'être appréhendé par les salariés dans leur expérience historique de son fonctionnement comme un faux dialogue. Le management participatif n'empêche pas les concepteurs d'avoir déjà un projet extrêmement avancé qui n'est susceptible d'amendements qu'à la marge et de le persévérer dans son application quoi qu'il advienne. Au contraire, la simulation peut éventuellement obliger les managers à se prononcer, à éviter qu'ils n'esquivent. Ce point prend d'autant plus sa valeur que, encore une fois, on a à l'esprit les réalités organisationnelles du participatif : dans le régime habituel de la conduite du changement, si les managers de premier niveau peuvent se dérober, ne pas donner de réelles suites à ce que les salariés proposent, c'est aussi parce que leurs propres managers ne veulent pas entendre la complexité qui leur est ainsi remontée. Ceux-ci renvoient les premiers vers le simple impératif de réexpliquer à leurs équipes et de faire passer le nouveau cadre d'activité quoi qu'il arrive. La simulation arme le manager de premier niveau dans son dialogue avec sa propre hiérarchie. 


\section{Conclusion}

24 changement plus authentiquement participatives que ce n'est au fond souvent le cas.
Resterait alors à évoquer les conditions de possibilité dans les organisations d'aujourd'hui. La question est énorme. D'un côté, une fenêtre d'opportunité existe avec le sentiment, profond ou non, des directions actuelles, de devoir, à cause de la digitalisation et/ou des attentes prêtées aux jeunes générations, faire une place à la mobilisation des intéressés dans la conception de ce qui les concerne. De l'autre, demeurent les rapports politiques de prise en compte du travail : l'organisation a, et gardera, bel et bien une préoccupation de contrôle et notamment de prévisibilité. Or, la prise en compte de l'activité complique assurément les choses par rapport à des modes de conception plus verticaux et instantanés qui ont l'avantage d'être, sur le coup, économiques.

Il y a donc une politique de la simulation. C'est par l'impulsion par les plus hauts niveaux de management que la simulation peut réellement exercer ses effets. En termes pragmatiques, dans une logique d'action au sein de l'entreprise ou d'intervention dans les organisations de toute nature, l'impératif est de travailler sur les managers de managers. Ce ne sont pas tant les membres du premier niveau d'encadrement qui pourront être embarrassés par la simulation. À ce niveau, on connaît l'activité. C'est leur hiérarchie, plus quotidiennement engagée dans le pilotage par indicateurs que dans la connaissance directe de l'activité qui peut poser problème. Elle détient, en effet, le pouvoir de refuser de libérer de l'espace pour le développement de la simulation et d'un point de vue sur l'activité, autant parce que cela la dérangerait que parce qu'elle peinerait à comprendre ce point de vue et à attribuer une signification à la simulation.

\section{BIBLIOGRAPHIE}

Banerjee, A.V., \& Duflo, E. (2009). The Experimental Approach to Development Economics. Annual Review of Economics, 1, p. 151-178.

Béguin, P., \& Cerf, M. (2004). Formes et enjeux de l'analyse de l'activité pour la conception des systèmes de travail. Activités, 1, n 1, p. 54-71.

Charles, J. (2016). La participation en actes. Paris : Desclée de Brouwer, 167 p.

Crawford, M.B. (2009). Eloge du carburateur. Essai sur le sens et la valeur du travail, trad. françaçse, Paris : La Découverte, 2010, 245 p.

Flichy, P. (2017). Les nouvelles frontières du travail à l'ère du numérique. Paris : Le Seuil, 420 p.

Ginsbourger, F. (2010). Ce qui tue le travail. Paris : Michalon, 187 p.

Gomez, P.-Y. (2013). Le travail invisible. Enquête sur une disparition. Paris : François Bourin Editeur, $254 \mathrm{p}$.

Activités, 15-1 | 2018 
Guérin, F., Laville, A., Daniellou, F. Durrafourg, J., \& Kerguelen, A. (1997). Comprendre le travail pour le transformer. Lyon : Editions de l'ANACT.

November, V., \& Creton-Cazanave, L. (dir.) (2017). La gestion de crise à l'épreuve de l'exercice EU Sequana. Paris : La Documentation française, $237 \mathrm{p}$.

Trupia, D.V. (2016). Produire un espace hybride de coopération. Une enquête ethnographique sur La Cantine. Réseaux, 196, 2016/2, p. 111-145.

Van Belleghem, L. (2012). Simulation organisationnelle : innovation ergonomique pour innovation sociale. $47^{e}$ congrès de la SELF, Lyon, 5-7 sept., 9 p.

\section{NOTES}

1. Ce qui suit emprunte à de multiples contributions au sein de cet ouvrage. Pour simplifier, je renvoie à l'ouvrage de manière collective et non à chacune des contributions mobilisées. Celles-ci ont principalement pour auteurs, outre les coordinatrices de l'ouvrage et dans l'ordre de leurs textes, S. Masson-Planchon, M. Reghezza-Zitt, J. Fayeton, S. Portier, V. Santoni, L. Audoin, S. Thomas, A. Adrot, M.-L. Sauvée.

\section{RÉSUMÉS}

L'article commente les contributions du dossier consacré à la simulation de l'activité. Se concentrant sur la simulation de l'activité en organisation, le commentaire défend que les méthodes correspondantes affectent potentiellement les rapports de pouvoir dans les processus de conduite du changement. Dans ces processus, les démarches participatives ne parviennent pas toujours à faire prendre en compte le point de vue de l'activité. Les méthodes de simulation s'attelleraient à faire prendre au sérieux les contraintes de l'activité et à envisager les ressources qui lui sont utiles.

The article discusses the contributions made to this issue on activity simulation. Focusing on the simulation of activity within organizations, it argues that the methods in question potentially affect power relations in change management processes. In these processes, participatory approaches do not always manage to take work activity into account. Simulation methods seek to take the constraints of the activity seriously and to consider the resources that would be useful.

\section{INDEX}

Mots-clés : simulation, activité, conduite du changement, management participatif Keywords : simulation, activity, change management, participative management 


\section{AUTEUR}

PASCAL UGHETTO

Université Paris-Est, LATTS

5, boulevard Descartes, Champs-sur-Marne, 77454 Marne-la-Vallée Cedex 2

pascal.ughetto@u-pem.fr 